\title{
King penguin brooding and defending a sub-Antarctic skua chick
}

\author{
W. Chris Oosthuizen ${ }^{1}$ and P. J. Nico de Bruyn ${ }^{1}$ \\ (1) Department of Zoology and Entomology, Mammal Research Institute, University of \\ Pretoria, Pretoria, 0002, South Africa \\ W. Chris Oosthuizen \\ Email:wcoosthuizen@zoology.up.ac.za
}

\section{Abstract}

Interspecific parental care is rare, conveys no ultimate evolutionary advantage, and is usually attributed to reproductive errors in species with analogous habitat, behaviour and diet. We report on interspecific parental care (brooding and "defence" of unrelated chick) provided by a king penguin to a sub-Antarctic skua chick on Marion Island, despite substantial risk of injury to the penguin due to the presence of the true parents.

\section{Introduction}

Interspecific alloparental care or adoption can be defined as parental care (brooding, defence or feeding) of nonlinear or unrelated offspring given by an individual replacing the original caregiver - usually a biological parent (Plissner and Gowaty 1988). This behaviour is rare and appears to conflict evolutionary theory, as kin selection is unattainable (Shy 1982). Such incidents have therefore usually been attributed to reproductive errors, where individuals fail to correctly identify offspring (Plissner and Gowaty 1988). We report on misdirected parental care by a king penguin (Aptenodytes patagonicus) in the form of brooding and "defence" of a sub-Antarctic skua chick (Catharacta antarctica) against the true parents.

\section{Observation}

On 22 December 2007, WCO observed a recently hatched (less than 7 days old) subAntarctic skua chick temporarily adopted by an adult king penguin of unknown sex at Goodhope Bay on sub-Antarctic Marion Island $\left(46^{\circ} 54^{\prime} \mathrm{S}, 37^{\circ} 45^{\prime} \mathrm{E}\right)$. Goodhope Bay is situated on the southern coastline of Marion Island and supports a breeding colony of approximately 840 king penguins (Crawford et al. 2003), which is divided into two subcolonies by a stretch of vegetated lava sands. The incident occurred on the vegetated area 
where no king penguins breed, but where they frequently rest. On arrival at the site two penguins were observed standing alongside each other, one of them brooding a chick. No king penguin chicks had hatched at this time. Closer inspection revealed that the penguin was brooding a sub-Antarctic skua chick and that two adult skuas, presumably the parents of the chick, were sitting in close proximity $(3 \mathrm{~m})$.

On return to the site an hour later the king penguin still brooded the skua chick. One skua repeatedly approached and harassed the penguin while also spreading its wings and calling, presumably in an attempt to regain its chick. On two occasions, it temporarily drove the penguin away from the chick (Fig. 1). The penguin defended itself and its adopted chick with pecking and flipper beats during the attacks, repositioning the chick on its feet after each attack in the typical posture of incubating/brooding king penguins (Fig. 2). The second skua parent remained in close proximity near the pair's other hatchling, which was hiding a few metres away (Fig. 3). No alloparental feeding was attempted in the observation period. Observations ended after 7 min when the king penguin pair was disturbed by a direct approach from another observer that was aimed at reuniting the chick with its parents. Both skua chicks successfully fledged later in the season.

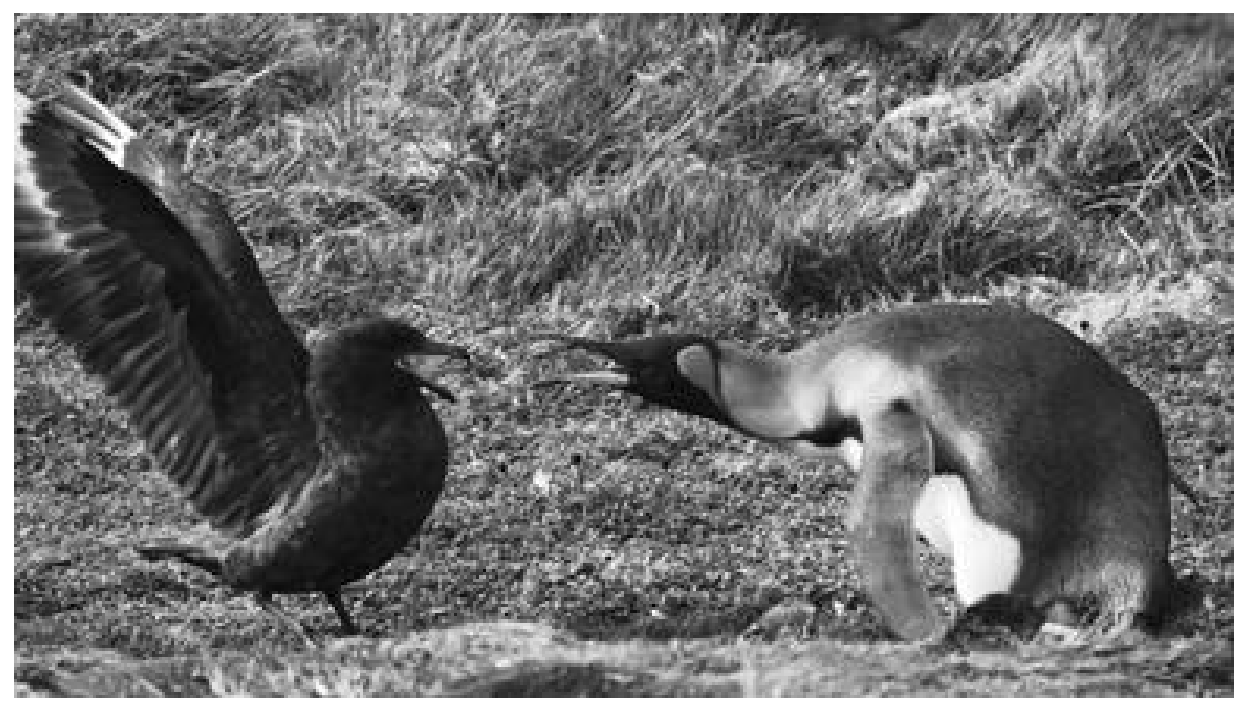

Fig. 1 The king penguin pecks at the attacking parent sub-Antarctic skua in defence of its adopted skua chick, visible on the ground in front of the penguin's flipper 


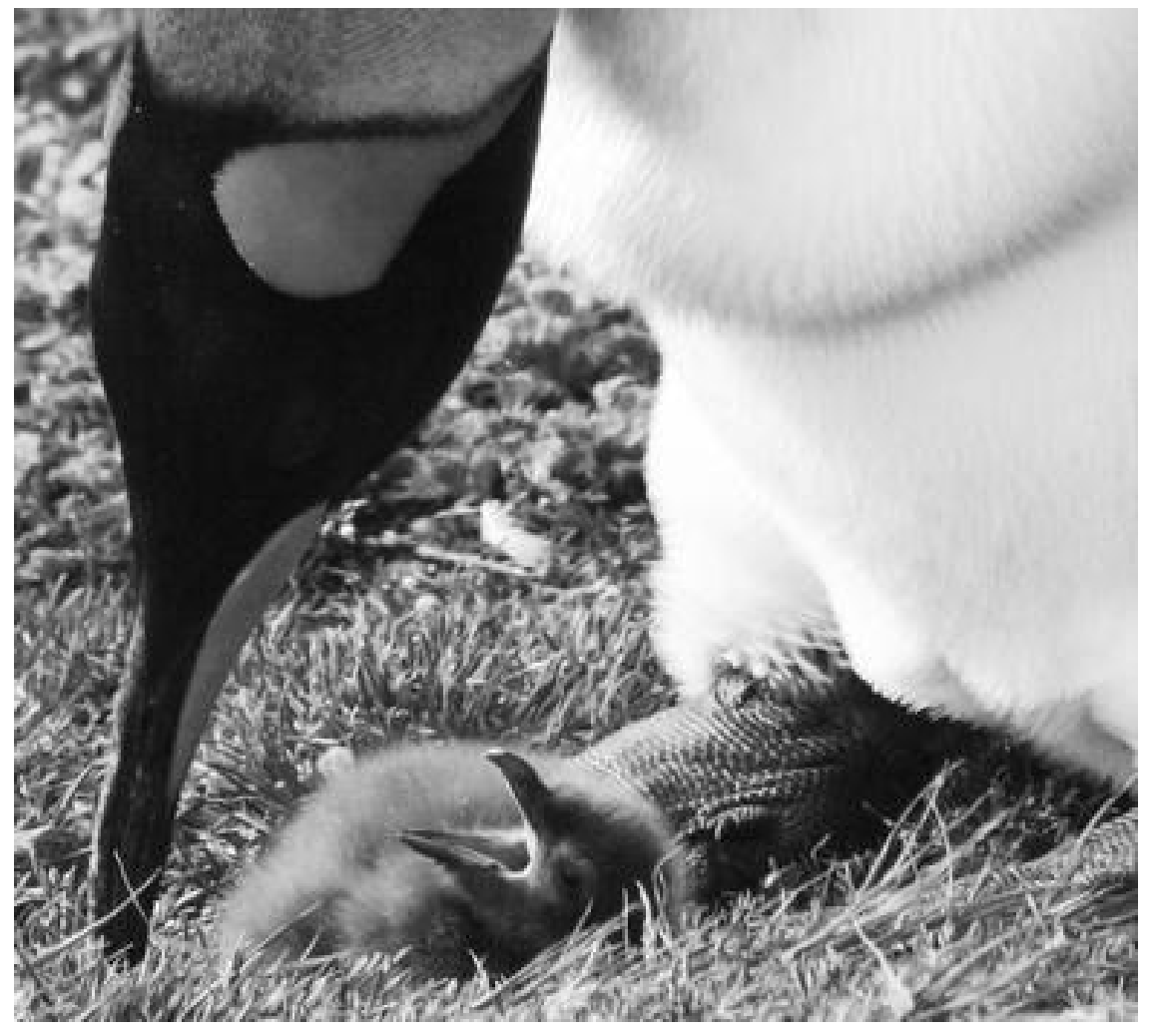

Fig. 2 The sub-Antarctic skua chick protests as it is hauled onto the penguin's feet in king penguin brooding style

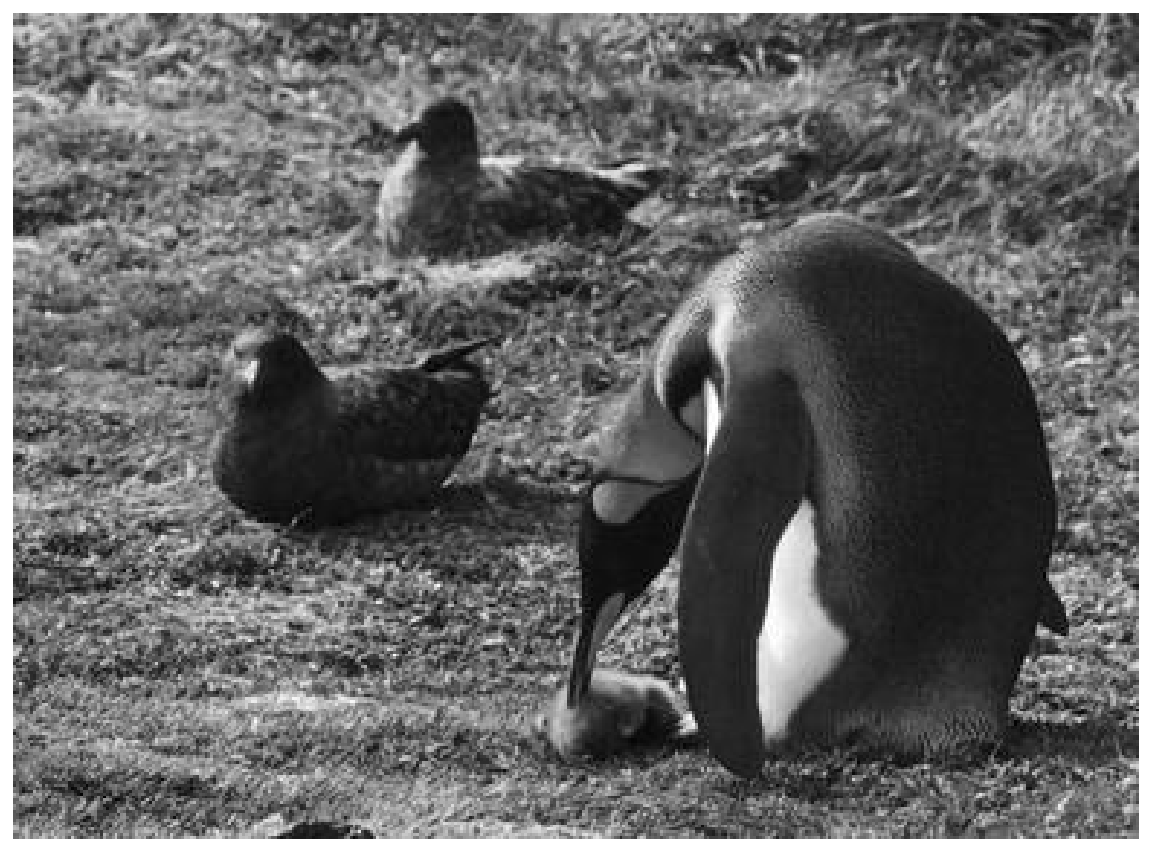

Fig. 3 The adopted sub-Antarctic skua chick is hauled into brooding position by the king penguin, with the skua parents in close proximity 


\section{Discussion}

To our knowledge, interspecific parental care amongst penguins or between penguins and other non-penguin species has not previously been reported. However, intraspecific parental care in king penguins is common and up to $25 \%$ of adults may allofeed (Lecomte et al. 2006). The association is short-lived, however, and parents do not repeatedly allofeed the same chick, apparently gaining no benefit from this behaviour. Emperor penguins (Aptenodytes forsteri) similarly allofeed chicks that are either found abandoned or are actively kidnapped (Jouventin et al. 1995). Although allofeeding was not observed in this case, the penguin brooded and defended (albeit against the chick's own parents) the temporarily adopted chick, actions associated with parental care. Most cases of interspecific parental care report on allofeeding, but Paz and Eshbol (2002) for example, observed common terns (Sterna hirundo) brooding and defending black-winged stilt (Himantopus himantopus) chicks from potential predators (and a possible biological parent).

Skuas predate on king penguin chicks and occasionally on adults (Hunter 1991). The penguin possibly failed to recognise the skua chick and considered it a king penguin chick that had been abandoned. Subsequently, it instinctively brooded and then defended itself and its adopted chick against the predatory skuas. An adaptive explanation for the adoption, where the penguin knowingly adopted a foreign chick, requires the penguin to gain some benefit from the adoption (e.g. demonstrating the ability to produce offspring or to lure potential mates for forthcoming breeding opportunities; Riedman 1982).

In both king and emperor penguin species, 'parenting hormone' prolactin may be endogenously controlled to maintain parental care during extended absences from the breeding site while foraging (Jouventin and Mauget 1996; Lormée et al. 1999). Elevated concentrations of prolactin have been linked to high intraspecific adoption rates in emperor penguins (Angelier et al. 2006). This observation, and others such as brooding of inanimate objects such as pebbles (personal observation), possibly support the view that prolactin may lower the ability of parents to resist brooding and parental care. However, in this case it remains speculative as no hormonal levels were measured. Although observations on the king penguin defending the chick were made for only a few minutes in the present study, the penguin brooded the skua chick for at least an hour prior to the observations and would have continued to do so for an unknown period if not for anthropogenic interference. The continued defence of a temporarily adopted, unrelated chick by the penguin, despite substantial risk of injury to itself during attacks by the skua, remains intriguing and may indicate hormonal levels overriding adaptive behaviour.

\section{References}

Angelier F, Barbraud C, Lormée H, Prud'homme F, Chastel O (2006) Kidnapping of chicks in emperor penguins: a hormonal by-product? J Exp Biol 209:1413-1420. 
Crawford RJM, Cooper J, Dyer BM, Greyling MD, Klages NTW, Ryan PG, Petersen SL, Underhill LG, Upfold L, Wilkinson W, De Villiers MS, Du Plessis S, Du Toit M, Leshoro TM, Makhado AB, Mason MS, Merkle D, Tshingana D, Ward VL, Whittington PA (2003) Populations of surface-nesting seabirds at Marion Island, 1994/95-2002/03. Afr J Mar Sci 25:427-440

Hunter S (1991) The impact of avian predator-scavengers on King Penguin Aptenodytes patagonicus chicks at Marion Island. Ibis 133:343-350.

Jouventin P, Barbraud C, Rubin M (1995) Adoption in the emperor penguin, Aptenodytes forsteri. Anim Behav 50:1023-1029.

Jouventin P, Mauget R (1996) The endocrine basis of the reproductive cycle in the king penguin (Aptenodytes patagonicus). J Zool (Lond) 238:665-678

Lecomte N, Kuntz G, Lambert N, Gendner J-P, Handrich Y, Le Maho Y, Bost C-A (2006) Alloparental feeding in the king penguin. Anim Behav 71:457-462.

Lormée H, Jouventin P, Chastel O, Mauget R (1999) Endocrine correlates of parental care in an Antarctic winter breeding seabird, the Emperor penguin, Aptenodytes forsteri. Horm Behav 35:9-17.

Paz U, Eshbol Y (2002) Adoption of black-winged stilt chicks by common terns. Wilson Bull 114:409-412.

Plissner JH, Gowaty PA (1988) Evidence of reproductive error in adoption of nestling eastern bluebirds (Sialia sialis). Auk 105:575-578

Riedman ML (1982) The evolution of alloparental care and adoption in mammals and birds. Q Rev Biol 57:405-435.

Shy MM (1982) Interspecific feeding among birds: a review. J Field Ornithol 53:370393 